\title{
Successful treatment of bepridil- induced intraoperative torsades de pointes by isoproterenol infusion
}

\author{
Ken Shimano, Kyungho Chang, Yoshiki Hara, Atsushi Yasuda and Shigehito Sawamura* (1)
}

\begin{abstract}
Background: Several types of antiarrhythmic drugs are known to induce QT prolongation and torsades de pointes.

Case presentation: An 84-year-old man was scheduled for open gastrectomy for residual cancer. He had been prescribed bepridil for atrial fibrillation that converted to sinus rhythm with prolonged QT interval in the operating room. After the surgery was initiated under general and epidural anesthesia, the patient's heart rate decreased to 50/ min and multifocal premature ventricular contractions appeared, followed by several episodes of torsades de pointes, each lasting for 5 to $15 \mathrm{~s}$. Infusion of isoproterenol was started $(0.01 \mu \mathrm{g} / \mathrm{kg} / \mathrm{min})$, and the heart rate was maintained at around $80 / \mathrm{min}$. Premature ventricular contractions disappeared, and torsades de pointes did not recur during the surgery. The operation was completed uneventfully. The serum bepridil concentration was found to be extremely high postoperatively.
\end{abstract}

Conclusions: Bepridil-induced intraoperative episodes of torsades de pointes were successfully treated by increasing the heart rate with isoproterenol.

Keywords: Bepridil, QT interval, Torsades de pointes, Isoproterenol, Bradycardia

\section{Background}

Apart from congenital causes, long QT syndrome can also be secondary to several medications including antiarrhythmics known to induce QT prolongation and torsades de pointes (TdP), termed the acquired long QT syndrome. Bepridil has been shown to convert atrial fibrillation (AF) to sinus rhythm (SR) in patients with persistent AF [1, 2]. Although marked QT prolongation and TdP reportedly occur in $2.8 \%$ and $0.9 \%$ of patients on bepridil treatment, respectively [3], there have been few reports describing intraoperative $\mathrm{TdP}$ induced by preoperative bepridil therapy.

*Correspondence: sawamura@med.teikyo-u.ac.jp

Department of Anesthesia and Critical Care, Teikyo University School

of Medicine, 2-11-1 Kaga, Itabashi, Tokyo 173-8606, Japan

\section{Case presentation}

Written informed consent was obtained from the patient for the publication of this case report and accompanying images.

An 84-year-old male was scheduled for open gastrectomy for residual cancer. He was prescribed with bepridil $(200 \mathrm{mg} /$ day $)$ and edoxaban $(60 \mathrm{mg} /$ day $)$ for AF. Electrocardiography (ECG) one month before the operation revealed AF with a heart rate (HR) of $122 / \mathrm{min}$ and QT/QTc interval of $324 / 396 \mathrm{~ms}$ (QTc: QT corrected with the Framingham formula, Fig. 1a). Echocardiography revealed an ejection fraction of $57 \%$ without wall motion abnormalities. He had no history of syncope. His renal and hepatic functions were normal. Edoxaban was withheld for 2 days before the surgery, while bepridil was continued until the morning of surgery to maintain control of $\mathrm{HR}$.

On admission to the operating room, ECG revealed normal SR with a HR of 72/min and QT/QTc interval 


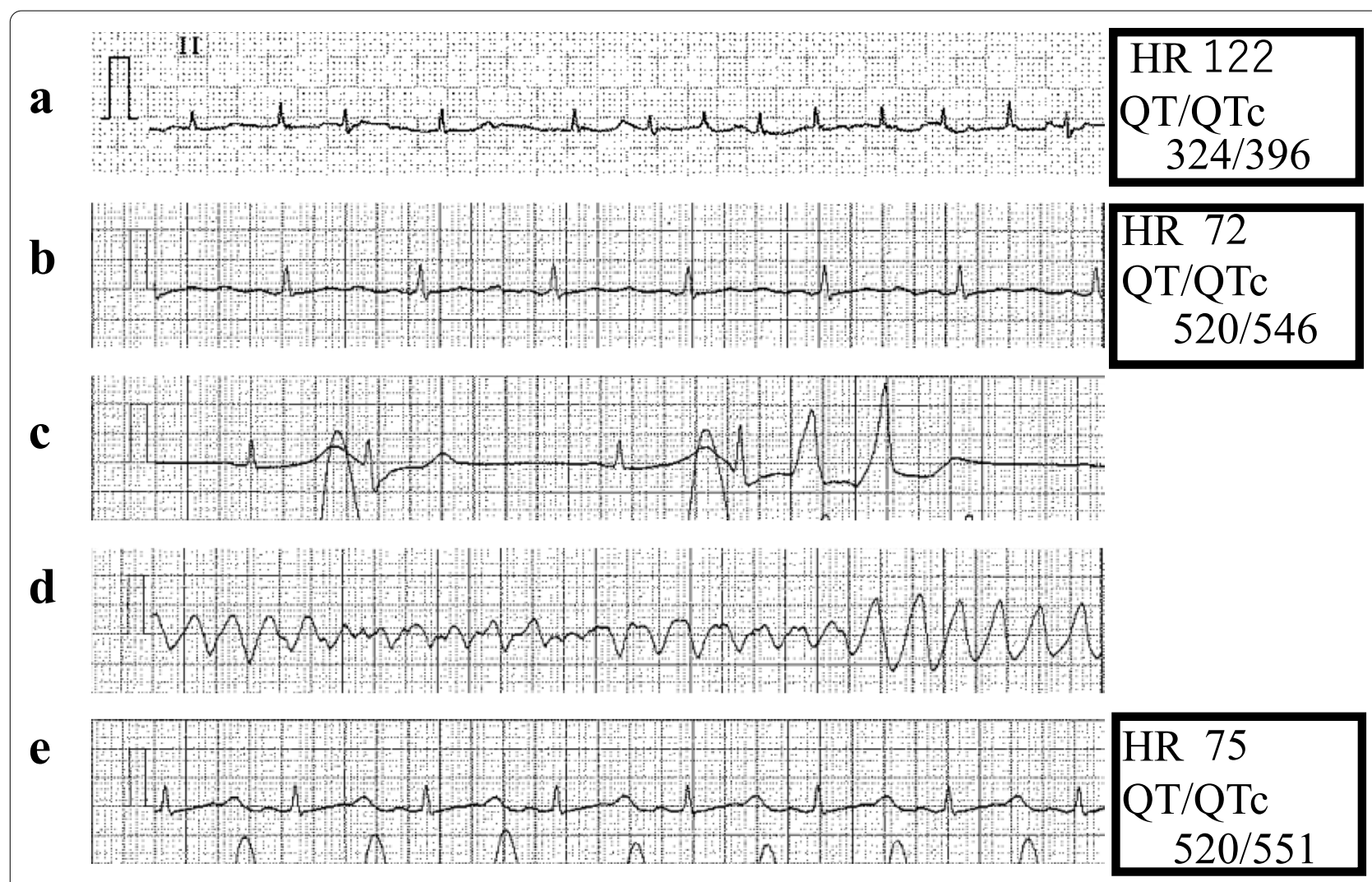

Fig. 1 Perioperative electrocardiographic changes. a One month before the operation: atrial fibrillation. $\mathbf{b}$ On admission to the operating room: sinus rhythm. c Eighteen min after the start of surgery: short runs of multifocal premature ventricular contractions (PVCs) are observed. $\mathbf{d}$ Twenty min after the start of surgery: torsades de pointes (TdP) lasting for $15 \mathrm{~s}$. e After the start of isoproterenol: sinus rhythm: PVCs and TdP have disappeared

of $520 / 546 \mathrm{~ms}$ (Fig. 1b). His blood pressure (BP) was $127 / 66 \mathrm{mmHg}$. A thoracic epidural catheter was inserted via the T7/T8 interspace. General anesthesia was induced with fentanyl $100 \mu \mathrm{g}$, propofol $80 \mathrm{mg}$, and rocuronium $50 \mathrm{mg}$ and maintained with remifentanil $0.1 \mu \mathrm{g} / \mathrm{kg} / \mathrm{min}$, sevoflurane $1.5 \%$, and rocuronium as required. Ropivacaine $(0.2 \%, 7 \mathrm{ml})$ was administered through the epidural catheter. After the administration of general and epidural anesthesia, his HR and BP gradually decreased (50/min and $90 / 40 \mathrm{mmHg}$, respectively). Eighteen minutes after the start of surgery, multifocal premature ventricular contractions (PVCs) and short runs were observed (Fig. 1c). Then, several episodes of $\mathrm{TdP}$, each lasting for 5 to $15 \mathrm{~s}$, occurred and terminated spontaneously (Fig. 1d). His systolic BP decreased to $30 \mathrm{mmHg}$, and pulse waves were not detected during TdP. The serum potassium level at this time was $3.6 \mathrm{mEq} / \mathrm{L}$.

Isoproterenol $(0.01 \mu \mathrm{g} / \mathrm{kg} / \mathrm{min})$ was started to increase the $\mathrm{HR}$ and counteract the QT-prolonging effect of bradycardia. A few minutes after the start of isoproterenol, once the HR increased to $80 / \mathrm{min}$, PVCs, short runs, and TdP disappeared and were not observed thereafter (Fig. 1e). Subsequently, potassium $10 \mathrm{mEq}$ and magnesium $20 \mathrm{mEq}$ were administered slowly.

The surgery was continued and completed as planned. The operation time was $4 \mathrm{~h}$ and $26 \mathrm{~min}$, and the blood loss was $130 \mathrm{ml}$. The patient was extubated uneventfully and admitted to the intensive care unit (ICU) with continued isoproterenol infusion. No life-threatening arrhythmias were observed in the ICU, though QT prolongation persisted.

The next morning, SR converted to AF with a HR of $120 / \mathrm{min}$ and QT/QTc interval of $235 / 333 \mathrm{~ms}$. Isoproterenol was discontinued, and carvedilol $(5 \mathrm{mg} /$ day) was started to control the HR. Four days later, AF again converted to SR with a HR of $89 / \mathrm{min}$ and QT/ QTc interval of 440/538 ms. No episodes of TdP were observed until discharge from the hospital.

Bepridil was not administered postoperatively. The serum concentrations of bepridil on the second and third postoperative days (2341 and $2415 \mathrm{ng} / \mathrm{mL}$, respectively) were found to be remarkably high with respect to the reported therapeutic range (250-800 ng/mL) [4]. 


\section{Discussion}

We report a case of intraoperative QT prolongation and $\mathrm{TdP}$ in a patient on bepridil. TdP was successfully treated by increasing the HR with isoproterenol.

Several medications, including antiarrhythmics, are known to induce QT prolongation and TdP, a rare but life-threatening arrhythmia. Johnston et al. analyzed 46 cases of perioperative TdP in the literature between 1978 and 2011 [5]. The main preceding events were QT prolonging drugs (30\%), hypokalemia (26\%), and bradycardia (15\%). The mean QTc at the time of TdP was $575 \mathrm{~ms}$.

Bepridil is indicated for conversion of AF to SR in patients with persistent AF $[1,2]$. However, bepridil reportedly also induces QT prolongation and TdP because of its multiple ion-channel blocking action, including that of potassium channels [3]. Careful followup is therefore highly recommended for the prevention of TdP during its use, especially in elderly patients. The risk factors for $\mathrm{TdP}$ in patients treated with bepridil include QT prolongation, bradycardia, hypokalemia, and advanced age [3].

QT/QTc interval on admission to the operating room was prolonged $(520 / 570 \mathrm{~ms})$ in our patient, though that measured 1 month before surgery was within normal limits $(324 / 396 \mathrm{~ms})$. It is known that QT/QTc intervals can fluctuate widely depending on the patient's activity, posture, circadian rhythm, and food intake. Therefore, we should keep in mind that preoperative assessment of QT intervals cannot always predict the intraoperative occurrence of TdP [6].

Conversion of AF to SR was related to QT prolongation in our patient. With the conversion of AF to SR, the QT/QTc changed from $324 / 396 \mathrm{~ms}$ to $520 / 546 \mathrm{~ms}$ preoperatively and from $235 / 333 \mathrm{~ms}$ to $440 / 538 \mathrm{~ms}$ postoperatively. It is not clear whether QT prolongation was caused by rhythm conversion itself or by decreased HR. There is one report describing TdP episodes soon after the conversion of AF to SR in patient on bepridil therapy [7].

Bradycardia is known to exacerbate QT/QTc prolongation and eventually trigger TdP in acquired long QT syndrome. During intraoperative management, opioids used for general anesthesia (fentanyl and remifentanil) and the sympathetic blocking properties of thoracic epidural anesthesia can often induce bradycardia. In fact, the HR decreased from 72 to $50 / \mathrm{min}$ after the induction of general and epidural anesthesia in our patient.

We should also pay attention to electrolyte abnormalities, especially hypokalemia and hypomagnesemia in patients with long QT. Hypokalemia $(3.6 \mathrm{mEq} / \mathrm{L})$ could have contributed to the occurrence of short runs and $\mathrm{TdP}$ in our patient, though they disappeared immediately after the start of isoproterenol infusion, that is, before the correction of hypokalemia.
Advanced age is reportedly associated with QT prolongation. Furthermore, sevoflurane reportedly causes greater QTc interval prolongation in elderly patients than in younger patients [8]. However, the extent to which sevoflurane increases the risk of life-threatening arrhythmias in patients with acquired long QT syndrome remains unclear.

Assessment of serum concentration of bepridil can be useful to predict the occurrence of TdP, because the QTc interval is reportedly significantly associated with serum bepridil concentration [4]. In our patient, serum bepridil concentrations measured on the second and third postoperative days were remarkably high with respect to the reported therapeutic range, suggesting that intraoperative QT prolongation and TdP were induced by bepridil.

Thus, preoperative evaluation of the risk of lifethreatening arrhythmias is crucial in patients on QT interval-prolonging drugs. Preoperative withdrawal of bepridil should have been considered in our case, though its serum concentration reportedly persists for several weeks after cessation [6]; the serum concentration of our patient did not change for one day (2341 vs. $2415 \mathrm{ng} / \mathrm{mL}$ ).

As bradycardia triggers QT prolongation and TdP in acquired long QT syndrome, increasing the HR could be a rational treatment strategy. In our patient, increasing HR pharmacologically with isoproterenol showed immediate and remarkable effects in the treatment of bepridilinduced TdP, as has been previously reported in an ICU patient [6]. As isoproterenol can be started quickly, we think it is worth trying in the operating room. Cyclic AMP amplification by isoproterenol can activate potassium channels and counteract QT prolongation caused by bepridil. However, the QT interval did not change after isoproterenol infusion in our patient. Temporary pacing could also have been effective in increasing the HR, but we did not try it because percutaneous pacing can often fail and the preparation for trans-jugular pacing can be time-consuming.

Lastly, although we chose isoproterenol treatment to increase the HR and counteract the QT-prolonging effect of bradycardia, bolus administration of magnesium could also be considered as an immediate treatment for TdP.

\section{Conclusions}

QT prolongation and repeated episodes of $\mathrm{TdP}$ were observed during combined general and epidural anesthesia in a patient on bepridil therapy. TdP was successfully treated by pharmacologically increasing the HR with isoproterenol infusion. 


\section{Abbreviations}

TdP: Torsades de pointes; AF: Atrial fibrillation; SR: Sinus rhythm; ECG: Electrocardiography; HR: Heart rate; BP: Blood pressure; PVC: Premature ventricular contraction; ICU: Intensive care unit.

\section{Acknowledgements}

We would like to thank Editage (www.editage.com) for English language editing

\section{Authors' contributions}

$\mathrm{KS}, \mathrm{SS}$, and KC performed perioperative management of the patient. KS collected the patient data. KS and SS wrote the manuscript. AY and $\mathrm{YH}$ revised the manuscript. All authors read and approved the final manuscript.

\section{Funding}

Not applicable

\section{Availability of data and materials}

Not applicable

\section{Declarations}

Ethics approval and consent to participate

This case report was exempted from institutional review board approval.

\section{Consent for publication}

Written informed consent was obtained from the patient for the publication of this case report and accompanying images.

\section{Competing interests}

The authors declare no competing interests.

Received: 18 August 2021 Revised: 18 September 2021 Accepted: 20 September 2021

Published online: 13 October 2021

\section{References}

1. Nakazato Y, Yasuda M, Sasaki A, lida Y, Kawano Y, Nakazato K, et al.

Conversion and maintenance of sinus rhythm by bepridil in patients with persistent atrial fibrillation. Circ J. 2005;69:44-8. https://doi.org/10.1253/ circj.69.44.

2. Yamashita T, Ogawa S, Sato T, Aizawa Y, Atarashi H, Fujiki A, et al. Doseresponse effects of bepridil in patients with persistent atrial fibrillation monitored with transtelephonic electrocardiograms : a multicenter, randomized, placebo-controlled, double-blind study (J-BAF Study). Circ J. 2009;73:1020-7. https://doi.org/10.1253/circj.cj-08-1061.

3. Yasuda M, Nakazato Y, Sasaki A, Kawano Y, Nakazato K, Tokano T, et al. Clinical evaluation of adverse effects during bepridil administration for atrial fibrillation and flutter. Circ J. 2006;70:662-6. https://doi.org/10.1253/ circj.70.662.

4. Matsui K, Mukai Y, Sakakura K, Wada K, Nakamura T, Kawabata A, et al. Relationship between serum bepridil concentration and corrected QT interval. Int J Clin Pharmacol Ther. 2021;59(1):63-70. https://doi.org/10. 5414/CP203843.

5. Johnston J, Pal S, Nagele P. Perioperative torsade de pointes: a systematic review of published case reports. Anesth Analg. 2013;117(3):559-64. https://doi.org/10.1213/ANE.0b013e318290c380.

6. Sonobe S, Imashuku Y, Yoshima Y, Sukenaga C, Inamori M, Yubata K, et al. Successful use of isoproterenol for bepridil hydrochloride-induced torsades de pointes. J Jpn Soc Intensive Care Med. 2014;21:521-2.

7. Shiraishi H, Kuroyanagi A, Shirayama T, Nakamura T, Yamano T, Matsumuro A, et al. A case of transient torsades de pointes after sinus conversion with bepridil administration for persistent atrial fibrillation. Shinzo. 2011;43(6):754-9. https://doi.org/10.11281/shinzo.43.754.

8. Nakao S, Hatano K, Sumi C, Masuzawa M, Sakamoto S, Ikeda S, et al. Sevoflurane causes greater QTc interval prolongation in elderly patients than in younger patients. Anesth Analg. 2010;110(3):775-9. https://doi. org/10.1213/ANE.0b013e3181cde713.

\section{Publisher's Note}

Springer Nature remains neutral with regard to jurisdictional claims in published maps and institutional affiliations.

\section{Submit your manuscript to a SpringerOpen ${ }^{\circ}$ journal and benefit from:}

- Convenient online submission

- Rigorous peer review

- Open access: articles freely available online

- High visibility within the field

- Retaining the copyright to your article

Submit your next manuscript at $\boldsymbol{\nabla}$ springeropen.com 